\title{
Integrating SNMP and CMIP alarm processing in a TMN management environment
}

\section{F. Munoz-Mansilla}

Professor at the University of Alcala de Henares (Spain) J.Sanchez.University of La Coruna (Spain).Nowadays at UCLA. V. Carneiro. Professor at the University of La Coruna (Spain). J. Coego. University of La Coruna (Spain). C/ Almirante Francisco Moreno, 5 bajo. 28040 Madrid. Spain. Ph.:+34 14590002 Email:\{fmm,jagrelo,victor,javier\}@cesat.es

\begin{abstract}
In a TMN management environment where SNMP and CMIP alarms coexist, CMIP notifications comply with TMN standards and provide complete information, whereas SNMP traps provide much less information, deviating from TMN standards. In order to handle this situation and make an homogeneous processing of alarms we propose an adaptor in charge of receiving any type of alarm and forwarding it in the same format (recommendation X.721 for the alarm types defined in X.733) independently of its original protocol. Besides this purpose, alarm contents are enhanced using additional information maintained in a repository.

The adaptor allows to model the required additional information. When a CMIP alarm is received the adaptor completes the information it carries. In the case of a SNMP alarm, the adaptor would integrate the trap contents in the TMN architecture (mediation device function, MDF). We include other TMN processes such as historic reporting of management data or alarm filtering and correlation.

An experimental prototype has been implemented using a management platform which allows shared access to information and distributed alarm management.
\end{abstract}

\section{Keywords}

Network management, alarm processing, SNMP, CMIP, TMN. 